\title{
Techno-economic evaluation of the island based introduction of optical cross connects in an IP-over-WDM network
}

\author{
Sofie Verbrugge • Didier Colle • Mario Pickavet • \\ Piet Demeester
}

Received: 27 June 2006 / Revised: 21 September 2006 / Accepted: 29 September 2006 / Published online: 9 December 2006

(C) Springer Science+Business Media, LLC 2006

\begin{abstract}
This article compares several traffic grooming approaches, based on expected capital expenditures (capex) to dimension and expand the network. For small traffic demands, the link-by-link grooming approach (efficiently filling SDH frames) is most interesting. We illustrate that, as traffic increases, there comes a point where the savings in IP layer expenses realized by endto-end grooming compensate the extra expenses of introducing the needed optical cross connects (OXCs). We study the network-wide migration from link-by-link towards end-to-end grooming at a single point in time, as well as a possible migration path, where OXCs are introduced gradually in so-called end-to-end grooming islands. This approach can lead to important savings in capex. We study total equipment costs as well as incremental and cumulative costs, indicating the total expenses to be paid by the operator over a certain time. The use of the Net Present Value (NPV) technique is clarified. Finally, also the sensitivity of the obtained results to changes in the component costs is studied.
\end{abstract}

Keywords Optical cross connect · OXC · Traffic grooming $\cdot$ Techno-economic evaluation $\cdot$ Migration

\section{Introduction}

In the field of network planning, theoretical work was done in the late 80 s $[1,2]$ and based on that, several authors have studied different parts of the network-

S. Verbrugge $(\varangle) \cdot$ D. Colle · M. Pickavet · P. Demeester Department of Information Technology (INTEC),

Ghent University - IBBT, Sint-Pietersnieuwstraat 41, 9000 Ghent, Belgium

e-mail: sofie.verbrugge@intec.ugent.be planning problem, among which long-term planning [3]. In the changing telecom landscape, techno-economic evaluations are very important when performing longterm planning. During the past years, the Internet traffic has known an important growth, which is still going on [4]. With respect to the observed traffic growth in backbone networks, it is a challenge for a network operator to find the most economical way to transport this traffic. A popular way of working in this regard is the use of grooming. This means optimizing the resource usage in a multi-layer transport network e.g. efficiently packing low-capacity traffic streams into high-capacity optical channels in an IP-over-Optical network [5]. Another approach to lower the costs for the operator is to introduce optical cross connects (OXCs) in backbone nodes. Those OXCs allow switching huge amounts of traffic on the optical layer. Among other benefits [6] this allows to avoid high IP layer costs, leading to important equipment cost savings. However, since OXCs are still very expensive today, it is important for the operator to find the optimal introduction time.

In this article, a capital expenditures (capex) comparison will be made between a network with and without OXCs using the appropriate grooming approaches. Different from other grooming studies e.g. [7,8] we study the impact of a growing traffic demand and thus the need to expand the network. Several migration scenarios are studied and compared to one another, using investment decision techniques.

Section "Trade-Off between several grooming techniques" introduces the two extreme grooming approaches, link-by-link and end-to-end grooming, and explains the existing trade-off between them. It also indicates several possible migration paths to introduce OXCs in an initially link-by-link grooming network 
(without OXCs). In Section "Island-based OXC introduction", the island based OXC introduction is identified to be the most relevant of the considered migration scenarios and it is compared to the two extreme grooming approaches, in terms of equipment costs. Section "Long-term planning decisions" indicates how results for network planning and extension need to be interpreted in the context of long-term planning. Section "Case study" reports the results of a case study, applying the above-mentioned techniques to a pan-European network. In Section "Sensitivity on equipment cost" we perform a sensitivity analysis and indicate how the previously obtained results are influenced by the used cost figures. The article ends with conclusions and guidelines in Section "Conclusions and guidelines".

\section{Trade-off between several grooming techniques}

\section{Link-by-link grooming}

In the traditional link-by-link grooming approach, link capacity is used as efficiently as possible. In an IP-overWDM network with SDH/SONET framing, this way of working can lower the total SDH card expenses. When the SDH lines are filled more efficiently (with traffic originating from several source-destination pairs), fewer lines are needed to transport all traffic, which may result in serious savings in case of low traffic volume. An important benefit of this approach is the efficient usage of (line) capacity on the optical layer. It implies, however, that all SDH frames need to be 'unpacked' after transiting a single optical link. Those frames may contain some traffic terminating in the considered node and some other traffic that needs to be sent on to another destination node. Link-by-link grooming is explained in Fig. 1. Although OXCs are shown on the optical layer of this figure, the link-by-link grooming approach does not require their presence. Link-by-link grooming can also be used in a WDM point-to-point system without switching capabilities on the optical layer. If we assume the two traffic flows in the figure to be equal and their sum to be smaller than the capacity of one wavelength, we need only one wavelength between each pair of OXCs. On the IP layer we need plenty of interface cards: twice the capacity of an individual traffic flow in the first node, four times this capacity in the second node, three times in the third node and once in the last node.

\section{End-to-end grooming}

In the end-to-end grooming approach, a wavelength is dedicated to traffic originating from a single source-

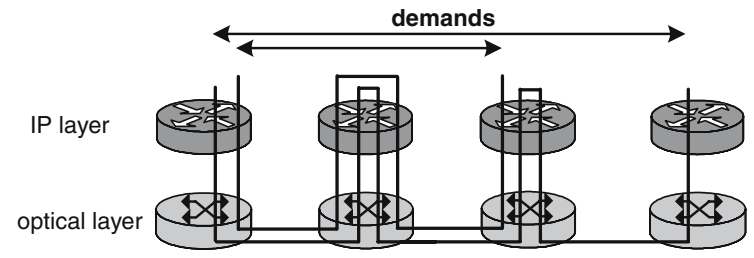

Fig. 1 Link-by-link grooming

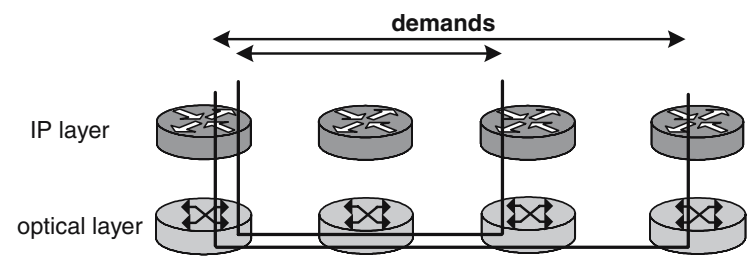

Fig. 2 End-to-end grooming

destination pair. This way of working is only possible if OXCs are present, allowing to set up an end-to-end light path. All transit traffic is bypassing the router optically, so that less router line cards are needed in case of large traffic volume, leading to an important saving in expenses on the IP layer. On the other hand, the optical line capacity is less efficiently used. An illustration is given in Fig. 2. Choosing between link-by-link and endto-end grooming therefore means making a trade-off between efficient use of line capacities and node costs. As long as the size of the individual traffic flows is smaller than the wavelength capacity (independent of the size of their sum), we need two wavelengths between the first and the third OXC and only one between the third and the fourth OXC. On the IP layer, we only need interface cards with the capacity of the two traffic flows in the first node and with the capacity of one traffic flow in the third and the fourth node. Compared to the example for link-by-link grooming in Fig. 1, more capacity is needed on the optical layer, less on the IP layer.

\section{Trade-off}

When making a trade-off between link-by-link and endto-end grooming, we need to consider both the expenses for the IP layer as well as for the optical layer. The expenses on the IP layer mainly consist of the SDH line cards. Due to transit traffic passing on the IP layer, these expenses are more important in case of link-bylink grooming. The expenses on the optical layer cost consist of the WDM line systems (mux/demux, optical amplifiers and transponders) and the OXCs. As each wavelength carries one SDH frame, more WDM line systems and more transponders will be needed in case of end-to-end grooming, leading to higher expenses on the 


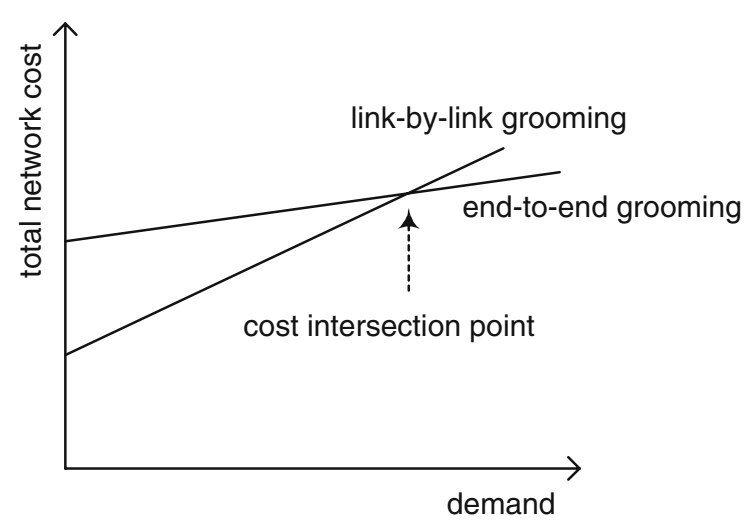

Fig. 3 The cost intersection point

optical layer. Moreover, in case of link-by-link grooming no OXCs need to be present in the network (and, indeed, they are not present in most of today's networks). They have to be introduced into the network especially in order to enable the use of end-to-end grooming. Remark that the "unit cost" of an OXC (to be paid at introduction time) is significant compared to that for other network equipment. The cost per bit, however, will be smaller for OXCs than for other equipment.

Note that we use the term expenses to denote actual expenditures. For instance IP router expenses denote the fraction of the overall capex spent on IP routers. By cost, on the other hand, we mean the price the vendor charges for a component e.g. a single SDH line card. Cost is an input to our study, expenses an output. Expenses can therefore be calculated by the product of the number of installed components and the cost of this type, summed over all relevant component types.

It is clear from the above discussion that, for small traffic demands, the link-by-link grooming approach (efficiently filling SDH frames) is most interesting. As traffic increases, however, the gain in IP layer expenses may compensate the high cost of introducing (OXCs) when using end-to-end grooming. The point in time where this happens will further be called the cost intersection point. From this point onwards (thanks to the growing traffic demand in the network) it will be more economical to use the end-to-end grooming than the link-bylink grooming approach. Link-by-link grooming is more interesting for small traffic demands, end-to-end grooming is more interesting for big traffic demands. The expenses to build a network according to the link-by-link grooming approach grow faster with the traffic demand to be routed over the network than the expenses to build a network according to the end-to-end grooming approach. A schematic illustration of the expected capex as a function of the demand is given in Fig. 3.
Migration paths

Network expansion is a typical dynamic and uncertain problem. The traffic will grow over time and the actual traffic demand for a future time period is unknown beforehand. Also the cost of the network equipment needed to dimension the network for this growing traffic is not constant, but will typically decrease over time. Based on uncertain forecasts for future traffic demands and component costs, the network planner needs to decide in which way the network will be expanded. This means deciding on future investments for several points in time. The decision may include migrating to another technology e.g. introducing OXCs. We have identified the following migration paths towards OXC introduction.

- Network-wide migration in a single step. In this case, we consider a link-by-link grooming network in which OXCs are introduced in all network nodes at a certain point in time and end-to-end grooming is used from that point onwards. The network is migrated from one extreme, link-by-link grooming (without OXCs) to another extreme, end-to-end grooming (with OXCs in all nodes). After this migration all traffic is routed directly from source to destination, without any special effort to fill the wavelength as well as possible. Critics may say that introducing OXCs will become interesting in the long run anyway (if we suppose that traffic will keep growing), and therefore suggest to introduce them right away. This way of working is not optimal, because of the equipment cost decrease and time value of investment. The OXC cost forms an important part of the equipment cost for a network operator using end-to-end grooming. If the OXCs are not needed right now, it may therefore be interesting to wait for the cost intersection point of Fig. 3. This point is determined by the cost evolution for a link-by-link and an end-to-end grooming network and (potentially) the transition cost between both approaches. Moreover, postponing an investment creates time value. If we can postpone an investment of e.g. $€ 1000$ for 2 years and we can still earn a yearly interest of $3 \%$ on it during those 2 years, we need less then $€ 1000$ today. The current value of the $€ 1000$ to be spent in two years is $€ 1000 /(1+3 \%)^{2}$.

- End-to-end grooming on a per source-destination pair basis. In the two extreme grooming situations (linkby-link and end-to-end grooming), the same grooming approach is used throughout the whole network in each period. However, it is also possible to use the end-to-end grooming approach for only some sourcedestination pairs. As the traffic is usually not uniformly 
distributed over the network, some source-destination traffic flows might be big enough to send them end-to-end, whereas others still need to be grouped to use the SDH frames efficiently. This approach requires that OXCs are available in those nodes over which the end-to-end traffic flows are routed. This way of working will reduce the IP layer expenses, because big traffic flows are sent end-to-end. On the other hand, an important cost is imposed by the requirement to have a lot of OXCs installed, namely on the path between all selected source-destination node pairs.

- Island based grooming. Another intermediate solution between link-by-link and end-to-end grooming is to introduce OXCs only in some nodes of the network. Those nodes are transformed into little islands where we use end-to-end grooming. Gradually more of these end-to-end grooming islands get introduced. An example is given in Fig. 4. In the beginning (step 1) link-by-link grooming is used throughout the whole network. If a certain node gets too heavily loaded, we install an OXC in that node, it becomes an end-to-end grooming island. In step 2 we notice one such node. As time passes, more of these islands get introduced and islands can be merged to become a bigger island. This is what we see in step 3, the island of the previous step has grown and a new one has appeared. Eventually (if the traffic keeps growing) the whole network can become end-to-end grooming.

The first migration path Network-wide migration in a single step compares two extreme situations and is thus very interesting as a reference scenario. On the other hand, it gives no flexibility to react on changes in the demand and cost. The intermediate solution of scenarios 2 and 3, on the other hand, allows the network planner to react on the dynamic and uncertain environment, by gradually introducing OXCs and migrating to end-to-end grooming. This means that the decision can be postponed a bit, till more information concerning actual traffic demands and equipment costs is available. We prefer scenario 3 Island based grooming over scenario 2 End-to-end grooming on a per source-destination pair basis as it allows to better spread the expenses for $\mathrm{OXC}$ introduction. In the remainder of this article, we will therefore only consider scenarios 1 (as a reference) and 3 (as a possible approach to gradually migrate the network).

Gradual migration leads to an intermediate situation between link-by-link and end-to-end grooming. It is important to notice, however, that this approach tries to find the best solution over the entire planning interval, whereas optimized grooming algorithms or heuris- tics $[9,7,8]$ aim at finding the best solution for a certain point in time (with a certain traffic). Those techniques consider a static situation. Some authors have studied the grooming problem with non-statistical dynamic traffic $[10,11]$. However, the study of network migration in terms of traffic grooming for dynamic traffic, growing over time, only received little attention so far. Our island based grooming approach considers growing traffic demand as well as changing component costs and studies the expected expenses over an entire planning interval.

\section{Island based OXC Introduction}

Impact on IP layer topology

In this section, we discuss the intermediate step in the migration from link-by-link grooming towards end-toend grooming, which allows to spread the expenses for OXC introduction. This way of working was introduced under the name island based grooming in the previous section. In those nodes where OXCs are introduced, the bypass traffic is sent on the optical layer, without going back to the IP layer, whereas in the other nodes all traffic still goes up to the IP layer. If the introduction of OXCs becomes beneficial in several adjacent nodes, those nodes can merge to a bigger island.

Figure 5 indicates how introducing an $\mathrm{OXC}$ in the optical layer changes the IP layer topology. All nodes adjacent to the node $B$ on the IP layer corresponding to the introduced OXC $A$ on the optical layer are connected by a direct IP link. Between the $n$ neighbours of node $B$, after the introduction of the OXC, there is a combination $^{1}$ of 2 out of $n$ direct links, see eq. 1.

$C_{n}^{2}=\frac{n !}{2 !(n-2) !}$

In the example of Fig. 5, $B$ has 4 neighbours. Before the introduction of the OXC there is one direct IP link (between the two bottommost IP layer nodes in the figure), after the introduction there are $C_{4}^{2}=6$ direct IP links. If OXC introduction is beneficial in two adjacent nodes, the IP layer topology is additionally changed so that the two nodes can be bypassed optically at once. It is clear that, with an important amount of OXCs (end-to-end grooming nodes), the logical layer becomes densely meshed. With OXCs in all nodes, we end up with a full mesh.

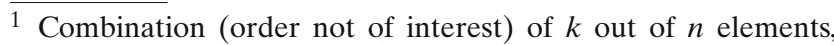
without repetition: $C_{n}^{k}=\frac{n !}{k !(n-k) !}$.
} 


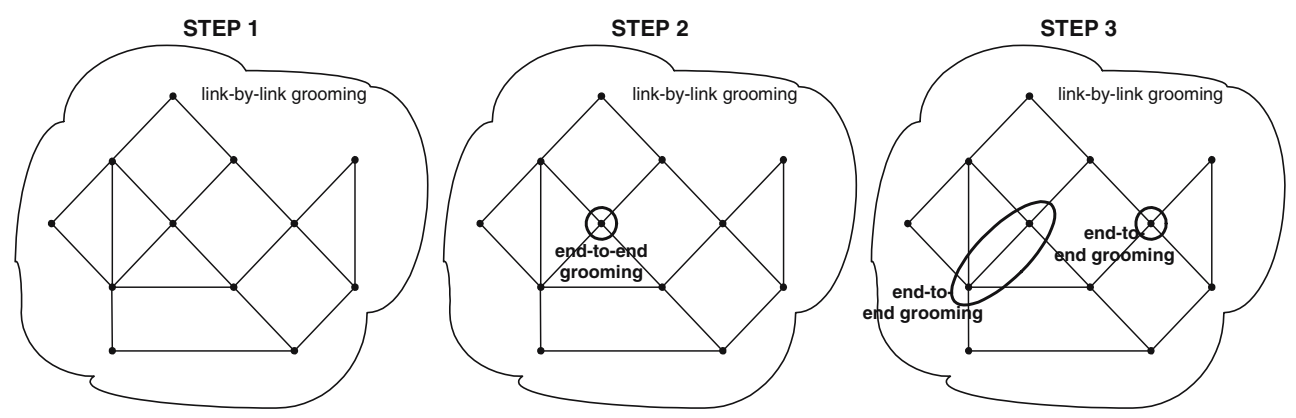

Fig. 4 Introduction of end-to-end grooming islands in link-by-link grooming network
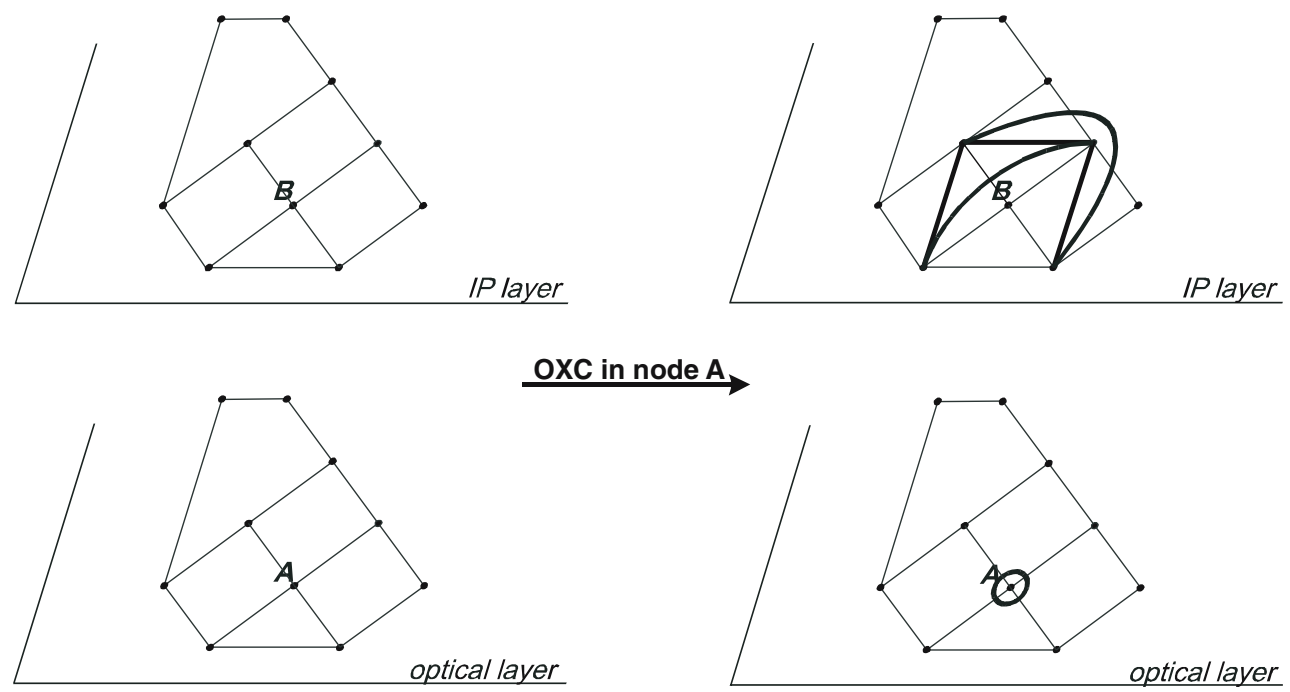

Fig. 5 Introduction of an OXC in the optical layer and its impact on the IP layer topology

\section{Cost model}

In order to decide if the introduction of an $\mathrm{OXC}$ is beneficial in a certain node, we base ourselves on a cost comparison for both solutions. The cost model we use in order to calculate the impact on the costs of the different network layers, is described below.

Several types of costs can be observed in the considered network, see Fig. 6. First of all, there is the IP layer cost, which consists of the unequipped IP router cost and the SDH line card cost. On the optical layer we differentiate between OXC cost, WDM line system cost and fibre cost. We consider an OXC that has an electrical switching fabric with short reach transponders (SR) on its interfaces. Those SR-transponders simply translate the electrical signal to an optical one, the nominal G.957 wavelength of $1310 \mathrm{~nm}[12]$, called $\lambda^{*}$ in Fig. 6. The WDM line system is composed of a WDM mux/demux with integrated optical amplifier and the necessary long reach transponders (LR). The latter are also called coloured transponders because they translate the nominal $\lambda^{*}$-signal into the desired wavelength (or colour: $\lambda 1, \lambda 2, \ldots$ ). We assume that transponders are installed on a per wavelength basis. This means that they are not physically integrated with the WDM mux/demux in some sort of transponder bank. Finally, there is the fibre cost, which consists of the physical fibre and optical amplifier cost. We assume that amplifiers are needed every $70 \mathrm{~km}$. Recall that the proposed cost model only considers capex. Operational expenses for actual operation of these components are not taken into account here.

For all components of the previously described model, a certain numerical cost is used. This cost, charged by the component vendor, is expressed as an unqualified number, relative to some base unit cost. The numbers used in this study are realistic cost figures, taken from the (partially confidential) cost model suggested by the IST-Lion project [13]. We have made some minor changes to this model. We differentiate between the cost of a SR and a LR transponder, assuming their cost ratio (SR/LR) to be 0.8 . In the total fibre cost, we do not take into account the cost of the physical fibre and the digging cost. In our model, fibre cost only consists of the optical 


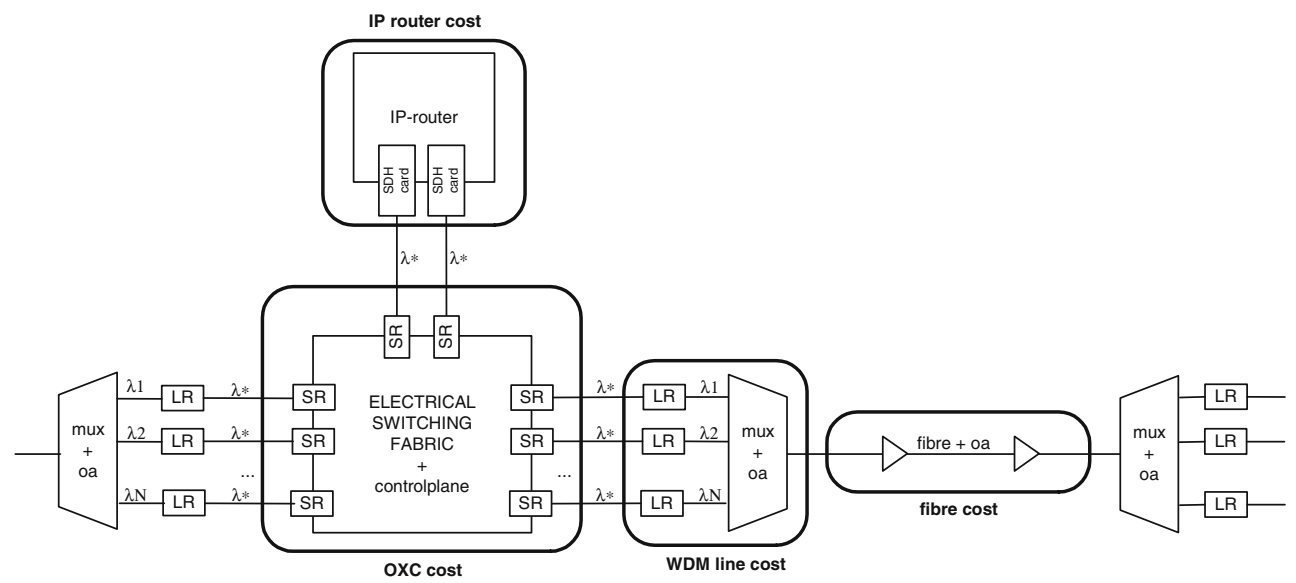

Fig. 6 Cost model

amplifier cost (every $70 \mathrm{~km}$ ). This assumption is based on the wide availability of dark fibre.

As mentioned before, in case link-by-link grooming is used, no OXCs are needed in the network. Therefore, the overall network costs in case of link-by-link grooming are given by the sum of the IP router cost (including SDH cards), the WDM line system cost (including LR transponders) and the fibre cost. In case of end-to-end grooming the overall network costs are the sum of these three costs plus the OXC cost (including SR transponder cost), because sending the traffic from source to destination on the optical layer is impossible without the presence of OXCs.

\section{Methodology}

Given this cost model, in the remainder of the article, we compare several grooming approaches on a basis of cost. When deciding on the introduction of an OXC in the network in the island based grooming approach, we base our decision on a local cost comparison between link-by-link and end-to-end grooming. Consecutively, we consider all nodes on the optical layer where no OXC is installed. We start from a pure link-by-link grooming network, so that, initially, no OXCs are installed. Therefore, we can start by randomly selecting a node on the optical layer. For the considered node, we dimension it using link-by-link and end-to-end grooming and compare the costs of both approaches. If the node is cheaper with the OXC (thanks to a reduction in the IP layer expense), the OXC is introduced, leading to an endto-end grooming island (as explained in the previous section). The procedure is described in Fig. 7.

When considering network planning over a longer planning interval (multiple planning periods, as described in the following section), the loop of Fig. 7 is executed for all nodes of the network (where no OXC has been installed so far) and the entire procedure (starting by selecting a first node) is repeated for every planning period.

\section{Long-term planning decisions}

When making strategic network planning decisions, a longer term planning interval (e.g. 5-10 years) will be taken into account. In that case, estimated equipment costs for several periods into the future, as well as total cost analysis over an entire planning interval will come into play. Those concepts are discussed in the current section.

Network equipment cost erosion

As time comes goes by, network equipment costs will typically decrease. The RACE Project TITAN [14] proposes some evolutionary trends for network component production costs versus their technological maturity. This model is based on a combination of an extended learning curve model (based on the Wright empiric law: "Each time the cumulated units production doubles, the unit cost decreases with a constant percentage") and a logistic growth curve model (where the market penetration volume is expressed as a function of time). Important parameters in this model are the observed component cost at reference time, the percentage of penetration volume at the reference time, the time it takes for the growth curve to go from $10 \%$ to $90 \%$ of the maximum penetration volume (viz. the time the product needs to be widely commercialized, $\Delta t$ ) and the relative decrease in the cost by doubling the production (learning curve rate or cost reducing factor $K$ ). Figure 8 


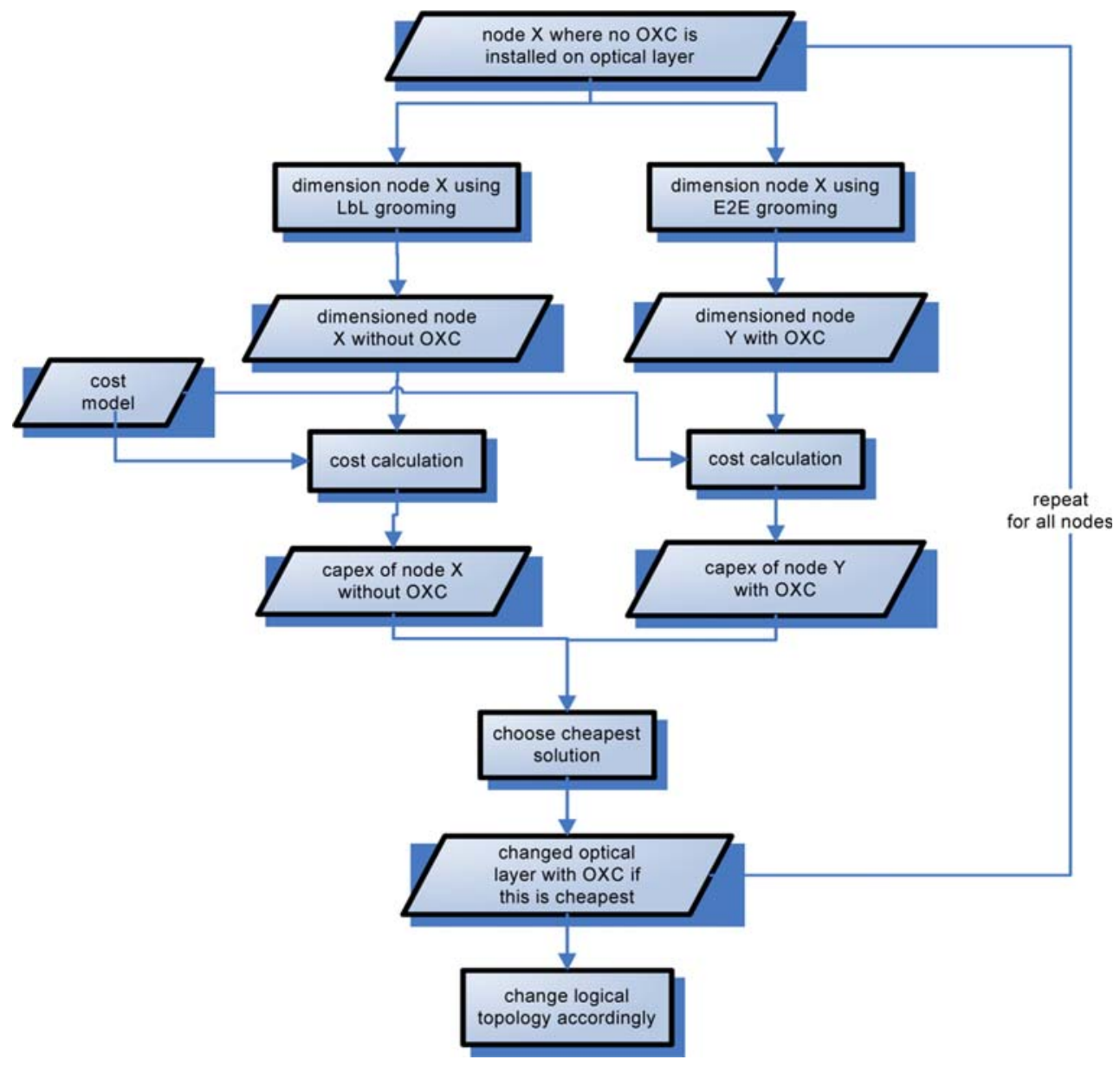

Fig. 7 Procedure to decide on OXC introduction

illustrates the meaning of the last two parameters. An important advantage of this model in the current fast evolving telecom environment is that it can also be used when only a few observations are available or if historical costs are absent. In the IST-Lion project realistic values were assigned to the parameters in this model [13], based on market experts' opinions and vendors' information. Those realistic equipment cost values are used in this case study.

Investment decisions based on costs for entire planning interval

When planning the expansion of his network, a network planner is interested in the expected expenses needed for this expansion over an entire planning period, rather than just for some future discrete time points. In order to compare expenses at several points in time in the future, the concept of the time value of money needs to be taken into account. This concept has been touched upon already briefly in Section "Migration paths". Therefore, cash flows spent on different time points need to be discounted to the same reference time in order to be comparable. This concept forms the base for the wellknown Net Present Value (NPV) investment decision technique [15]. Net Present Value compares the present value of several investment projects and chooses the solution with the highest present value. The present value of an investment project is defined as the sum of the expected cash flows $C_{n}$ over the economic lifetime of the project $N$, discounted with the expected rate of return $i$ (the discount rate). Equation (2) gives the formula to calculate the NPV. The used discount rate should reflect the expected return to be earned on the project. It grows with the amount of risk involved in the project. For a riskless project, it equals the interest rate that can be earned on a simple bank account. For a telecom project, a common interest rate is $10-15 \%$.

$\mathrm{NPV}=\sum_{n=0}^{N} \frac{C_{n}}{(1+i)^{n}}$ 
Fig. 8 Definition of parameters in extended learning curve model
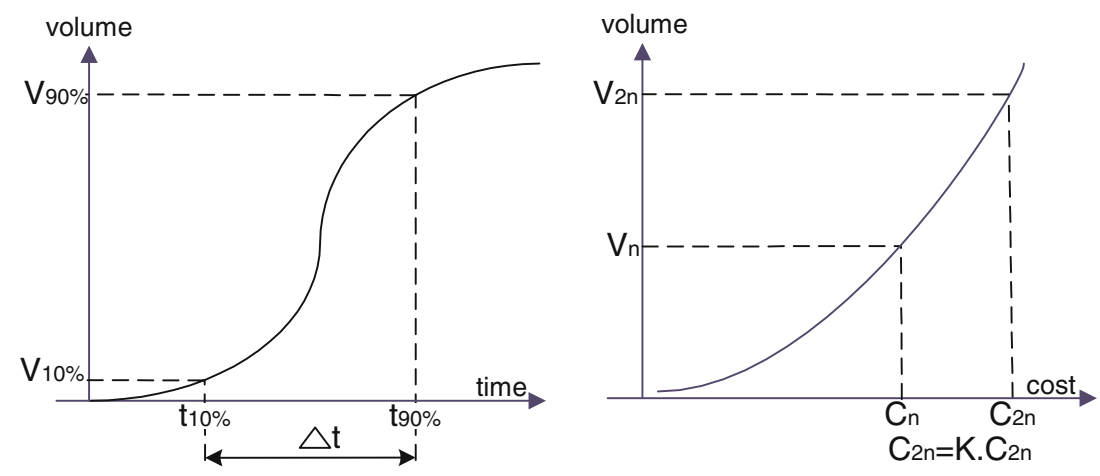

Remark that, apart from NPV, some older and less reliable decision criteria are still used in practice today (instead of or in addition to the use of NPV). Those include maximum initial investment cost (incremental investment for first time period: $C_{0}$ ), total cost of ownership (TCO, the total cost of the project over the entire life time, neglecting the income), or maximum (discounted) payback time (the time it takes till the expected revenues for a certain time interval compensate the cumulative equipment cost over that interval).

Below, we are going to study the following three network migration approaches:

- Capacity expansion using link-by-link grooming The network is expanded using link-by-link grooming. There are no OXCs installed.

- Capacity expansion by network-wide migration towards end-to-end grooming At migration time, OXCs are installed in all network nodes and from that point onwards end-to-end grooming is used throughout the whole network. Before migration time, there are no OXCs and the network is expanded using linkby-link grooming.

- Island based migration Starting from a link-by-link grooming network, OXCs are gradually installed in end-to-end grooming islands.

For the different migration approaches, we will compare the total costs to build to network to cope with the traffic in the different planning intervals. This is interesting for a new entrant into the market, trying to find the optimal approach when entering the market from a green field situation. However, for an incumbent operator already disposing of a network that is up and running, the total cost (to build the network from scratch) is not the most important parameter as a basis for his network expansion decisions. The incremental costs (needed investment per time period) to expand the network from the existing situation will be more relevant. When expanding an existing network, the incremental costs are exactly the cash flows that need to be considered when applying NPV as an investment decision technique.

\section{Case study}

\section{Definition}

We consider a pan-European IP-over-Optical network with 28 nodes and 41 links in its physical topology (base topology of [16]), see Fig. 9. In order to dimension the network over a time period including the current situation, we use the traffic estimated by the traffic volume forecast model first proposed by Vaughn and Wagner $[17,18]$ and applied to the pan-European network in Ref. [19] (model 2 of [19], with the reference data for year 2002 and an annual growth of $10 \%$ for voice traffic, $34 \%$ for transaction data traffic and $100 \%$ for IP traffic). This traffic model estimates the total panEuropean traffic. We assume that a big pan-European operator will be able to attract $30 \%$ of this traffic and therefore we dimension the network for $30 \%$ of the traffic predicted by the traffic forecast model. We study the time period 2004 till 2014. (All information concerning topology and traffic can be found in Ref. [19]).

We use the node model and the associated equipment cost model of Fig. 6. Only one size of a certain component type is considered. We assume that all SDH line cards have a capacity of $10 \mathrm{Gbps}$, the IP routers have a capacity of $200 \mathrm{Gbps}$, the WDM line systems take 40 wavelengths and the OXCs have 512 ports. When an IP router or an OXC is full (all capacity used), it needs to be upgraded by adding another router or OXC of the same size. Hereby we neglect the interconnection: if 512 ports do not suffice in a certain node, several identical OXCs are installed next to one another. In practice, however, more ports are needed for OXCto-OXC interconnection as the number of interconnected OXCs grows. The same goes for IP routers, as described in Ref. [20]. Several types of interconnection could be studied in order to minimize the number of 


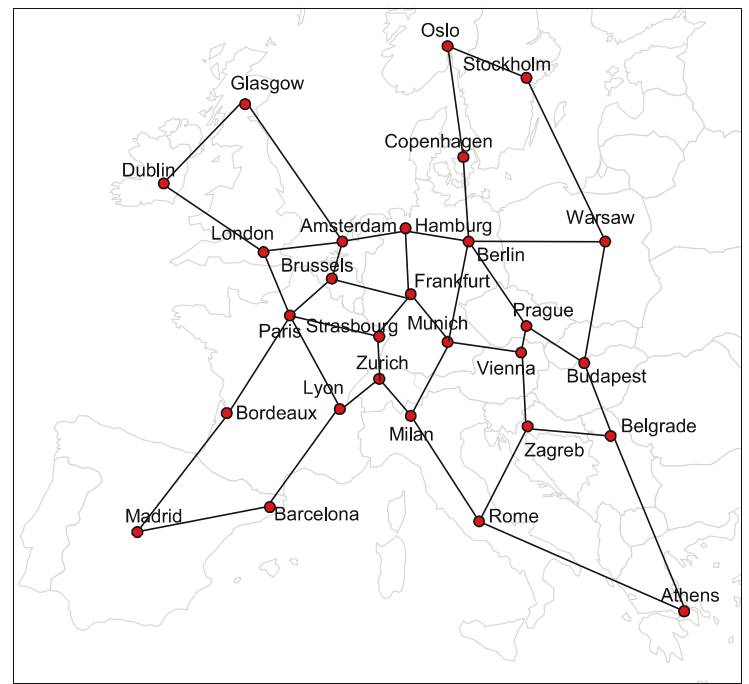

Fig. 9 Pan-European network under study

interconnection ports, but this is not considered here. We consider opaque OXCs (with an electrical core) so there is no problem of wavelength assignment and regeneration [21].

\section{Link-by-link versus end-to-end grooming}

For the traffic growth described above over the time period 2004-2014, the total costs to dimension the network according to the two extreme grooming approaches (link-by-link versus end-to-end), are shown in Fig. 10 (only plotted till 2012). We see that until 2008 the linkby-link grooming approach is most economical. As the demand is fairly small compared to the capacity of the considered SDH frames, efficiently filling those frames leads to an important gain. As the demand increases, the capacity of a SDH frame is better approximated by the demand for a single source-destination pair. The intersection point of the graphs is situated around late 2008 . This means that, for a new operator entering the market, it would be interesting to set up a network with OXCs and use end-to-end grooming right away from that time onwards. After 2009, end-to-end grooming is cheaper than link-by-link grooming, which means that the gain in IP layer expenses obtained by applying end-to-end grooming is able to compensate the additional expenses for introducing OXCs in all network nodes. Note that the end-to-end grooming graph decreases a bit between 2004 and 2008. Although the traffic grows in this interval, the overall capex to dimension the network decreases. This can be explained by the used decreasing equipment cost. In this interval, the equipment cost decreases faster than the growth of the traffic. Remind that the

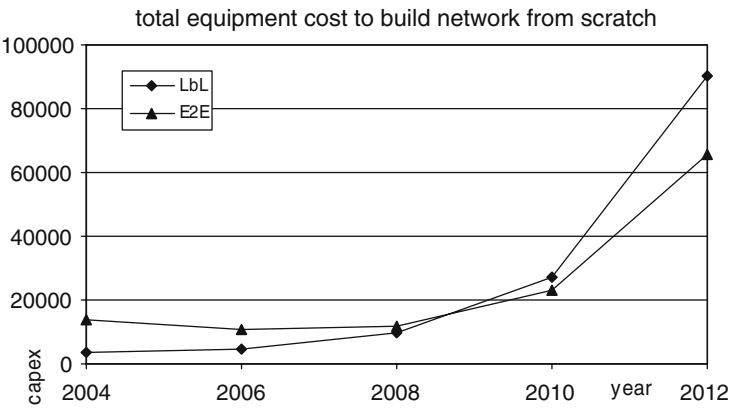

Fig. 10 Capex comparison for different grooming approaches, green field situation

yearly cost decrease is not constant, but rather given by the learning curve model.

It is clear that the total considered traffic demand has an important impact on the cost intersection point between link-by-link and end-to-end grooming. If the operator is able to attract more traffic than the $30 \%$ market share assumed above or if the traffic grows faster than predicted by the traffic model we used here, the intersection point will come sooner than 2009. In case of less traffic, the intersection point falls later. Ref. [22] shows that the intersection point for a monopolist operator (attracting all traffic) falls 2 years sooner than for an operator attracting only a $25 \%$ market share (using a slightly different cost model and a smaller network). This finding can be explained by the fact that annually doubling IP traffic (which constitutes the dominant traffic fraction) was assumed.

Figure 11 considers an operator with an existing linkby-link grooming network (dimensioned for the traffic of 2002) and indicates the incremental costs to expand this network, either using link-by-link grooming or using end-to-end grooming (and thus introducing OXCs in all nodes in 2004). The important investment of introducing the needed OXCs, can clearly be observed in 2004 in the curve for end-to-end grooming in Fig. 11. In subsequent time intervals, the incremental costs denote the cost of the additional equipment needed because of the observed traffic growth. These costs are lower in case of end-to-end grooming, because less expensive SDH line cards need to be installed in this case. The situation for an incumbent operator is studied in more detail in Section "Long-term planning decisions".

\section{Island based OXC introduction}

Figure 12 shows the nodes where OXCs are introduced in the pan-European network when considering island based OXC introduction as a way of gradually migrating from link-by-link towards end-to-end grooming. In 


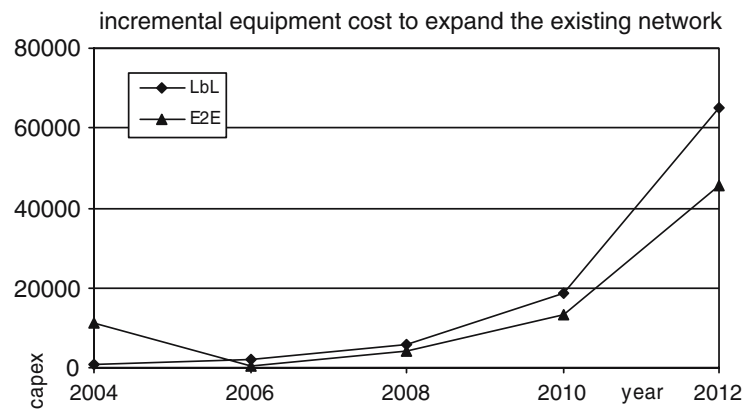

Fig. 11 Capex comparison for different grooming approaches, situation for incumbent

2004 , the most economical solution is to use link-by-link grooming in the entire network, without introducing any OXCs. With the traffic of 2000, it becomes interesting to bypass the traffic in some nodes in the core the network. Two end-to-end grooming islands appear: an end-to-end grooming island with 6 nodes containing Amsterdam, Hamburg, Berlin, Munich, Zurich and Milan and an isolated end-to-end grooming node in Paris. As the traffic grows further, more and more OXCs should be introduced. In 2008, the two islands have merged and grown to an island containing 13 nodes, whereas a new isolated end-to-end grooming node appears in Dublin. In 2010, the big island grows to 15 nodes and now also includes the new end-to-end grooming nodes in Bordeaux and Zagreb. In 2012, nearly all nodes (19) in the core of the network (no OXC in Prague) have OXCs and end-toend grooming is used in this entire domain. After this, the situation does not change very much any more. In 2014 also London enters the end-to-end grooming part of the network, so that the two islands merge to one. This brings the total number of end-to-end grooming nodes in the network to 20 . Only in 8 (mainly) edge nodes the transit traffic is not big enough to justify the introduction of an OXC. In those nodes, all traffic keeps passing through the IP layer. The fact that it takes so long till an OXC gets introduced in London can be explained because the used traffic model only considers European traffic. In London we expect an important amount of transatlantic traffic towards the United States, not considered here.

When we compare the capex (total equipment cost) to dimension the network using link-by-link, end-to-end and island based grooming, we find that island based grooming is indeed a good compromise between both extreme approaches. As there are no islands installed in 2004, the expenses for the island based grooming approach are equal to those for the link-by-link grooming approach. In this case, the gain which can be made compared to end-to-end grooming is $75 \%$, so that intro- ducing OXCs would definitely not be a good idea here. As the traffic grows, more islands are suggested by the island based grooming approach, so that the gain compared to end-to-end grooming (with OXCs in all nodes) decreases, towards $3 \%$ for 2014 ( 20 of the 28 nodes have OXCs). On the other hand, the gain to be made compared to link-by-link grooming increases with growing traffic, from $0 \%$ in 2004 to $34 \%$ for 2014 .

\section{Long-term planning decisions}

Considering the network-wide migration from link-bylink towards end-to-end grooming, the optimal migration time can be determined using the NPV technique. We consider the incremental costs over the time period 2004-2014 to expand the network according to the growing traffic demand. We consider several possible timings to perform the network-wide migration from link-bylink towards end-to-end grooming. (This extends the study of Fig. 11, where the migration in 2004 for an incumbent operator was already studied.) Fig. 13 indicates the NPV the sum of those incremental expenses ${ }^{2}$, discounted using a discount rate of $10 \%$. We observe that 2010 would be the best migration time from linkby-link towards end-to-end grooming (lowest NPV).

Both the green field situation and the situation for the incumbent are shown in the Fig. 13. We observe the same trends for both cases, the only difference is the cost in the first period (building the network from scratch in the green field situation and expanding the existing situation for the incumbent). This initial cost difference between the green field and the incumbent situation can be observed in the difference in height between the corresponding bars in the two graphs of Fig. 13.

An important parameter in the NPV formula is the economical life time of the project $N$. It indicates the time over which the investments can be used in an economical way. In case of long-term network planning, this should correspond to the planning interval. In Fig. 13 the planning period was set to 10 years, 2004-2014. In Fig. 14 the impact of a smaller planning interval is studied. We see that, for $N$ up to 4 years (considered interval 2004-2008 or smaller), three of the considered options

\footnotetext{
2 We have calculated the NPV based on the network equipment expenses to expand the network. To calculate the total NPV for the network operator, apart from expenses, also income for the operator needs to be counted in the considered cash flows. In our study, we have always dimensioned the network to be able to carry the entire demand (30\% of demand predicted by traffic forecast model). This means that the incomes are equal under all grooming scenarios. Neglecting them in the NPV calculations influences the actual $N P V$ values that are obtained, but does not influence the comparison between the approaches.
} 

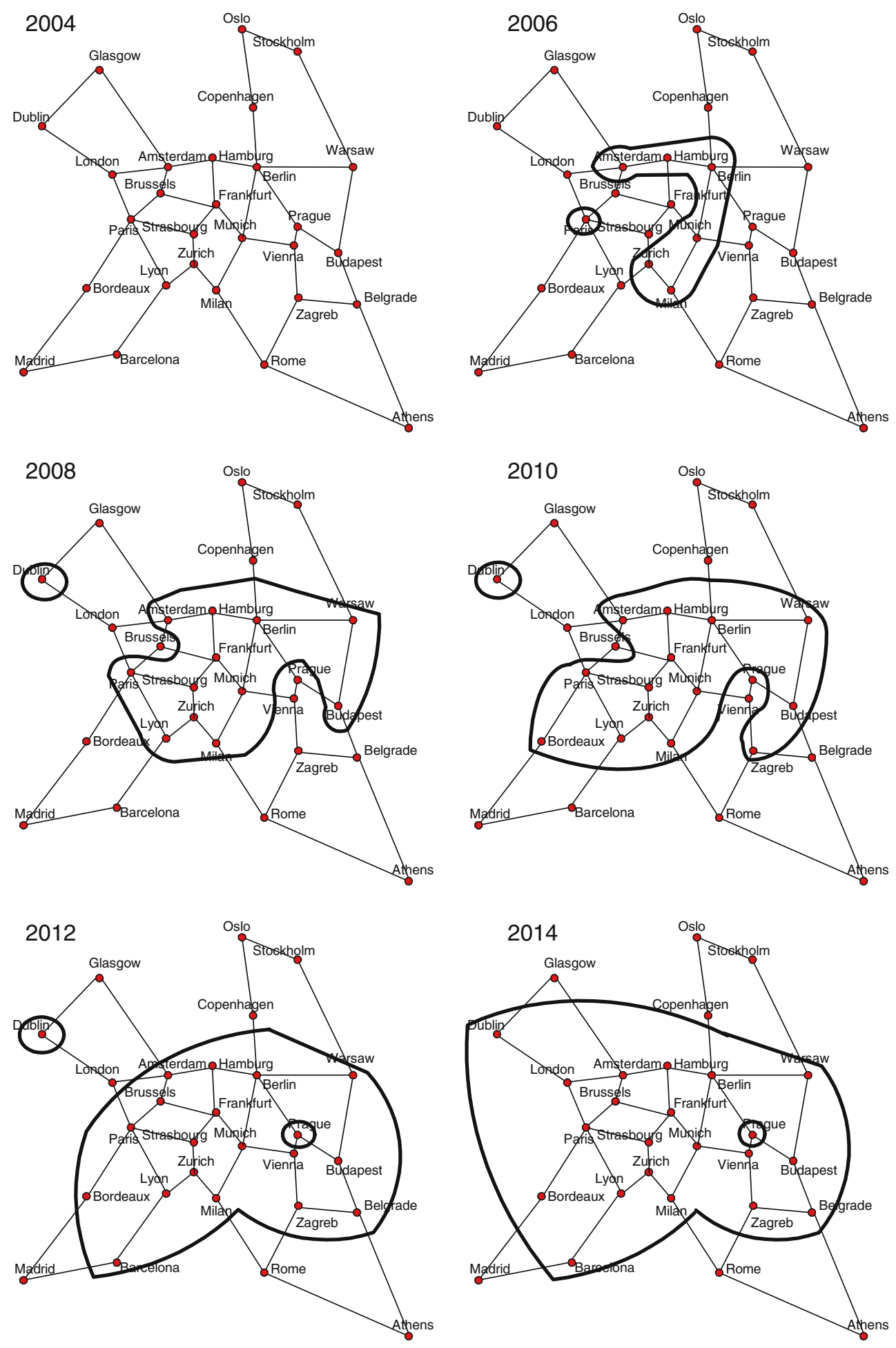

Fig. 12 Evolution of end-to-end grooming islands in pan-European network

have similar NPV: expansion using link-by-link grooming, island based migration and network-wide migration in 2010 (which is actually equal to link-by-link grooming in the considered interval). Network-wide migration in 2004, leads to significantly higher NPV and therefore needs to be avoided. When considering a planning interval of 6 years (2004-2010), it becomes clear that network-wide migration in the year 2010 is a better option than continuing to keep expanding the network using link-by-link grooming. Island based grooming is clearly the best option. Enlarging the planning interval even further, makes clear that migration towards end-to-end 
Fig. 13 Determination of optimal migration time from link-by-link towards end-to-end grooming
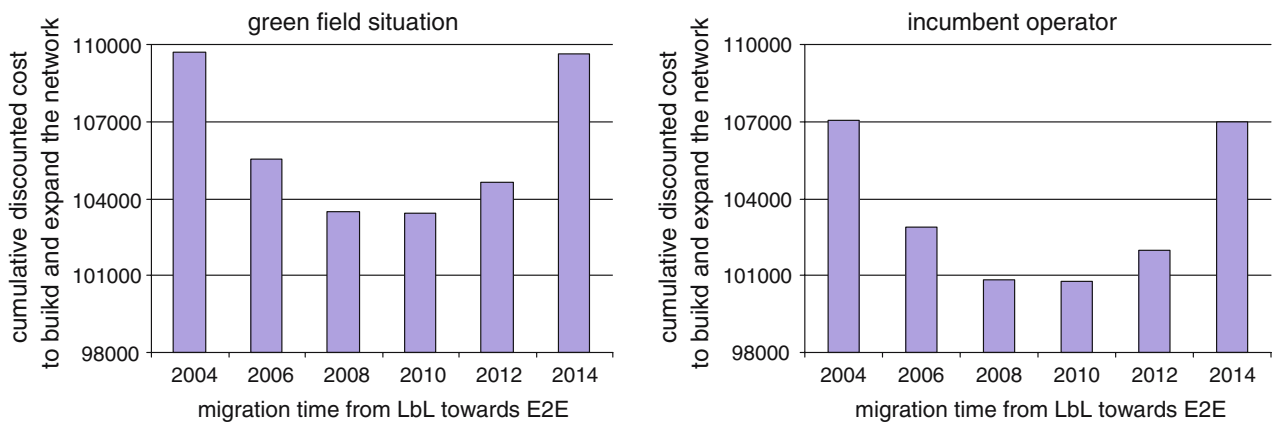

Table 1 Impact of component cost decrease on cost intersection point

\begin{tabular}{ll}
\hline Component with decreased price & Cost intersection point \\
\hline None & July 2008 \\
Wdm mux & July 2008 \\
Optical amplifier & July 2008 \\
SDH line card & January 2011 \\
Transponder (SR and LR) & October 2007 \\
Unequipped OXC & June 2008 \\
\hline
\end{tabular}

grooming definitely is to be preferred over the continued use of link-by-link grooming. With a planning interval of 8 years (2004-2012), network expansion based on link-by-link grooming is the worst option. Even immediate migration towards end-to-end grooming (in 2004) is better.

\section{Sensitivity on equipment cost}

As mentioned before, in our case study the cost figures (decreasing over time) suggested by Ref. [13] were used. In this section, we want to find out the sensitivity of the overall expenses to the equipment cost figures. Therefore, we will change the cost per component type. Table 1 shows the cost intersection point between link-by-link and end-to-end grooming if the cost for a certain component type is changed to half of its original cost, while the cost of the other components is left unaffected. It was shown before [23] that this approach leads to similar sensitivity results as the use of a cost decrease over time for the considered component type.

The cost intersection point found above for the equipment costs of [13], was July 2008. The sensitivity to changes in the WDM mux cost is very low, the capex intersection point remains unchanged. The same goes for changes in the cost of the optical amplifiers. If the cost of a SDH line card were half of what is expected by the model of [13], link-by-link grooming would stay the cheapest approach till January 2011. If the transponder cost (SR and LR) were half of its original, the end-to-end grooming approach (requiring a lot of transponders) would be favorised sooner (from October 2007 onwards). Lowering the cost of an unequipped $\mathrm{OXC}$, finally, slightly favours the end-to-end grooming approach, leading to a capex intersection point in June 2008. Remark that the cost ratio SR transponder/ LR transponder is kept constant (at 0.8) throughout this study. We believe this to be a realistic assumption. However, some previous studies [23] have shown that the sensitivity of the overall expenses to changes in this ratio is big.

The impact of the SDH line card cost on the cost intersection point can easily be understood from the importance of this cost in case of link-by-link grooming, see the left most part of Fig. 15. In case of big traffic demands (2006 and later), the SDH line card cost definitely is the dominant factor in the link-by-link grooming expenses. Reducing this cost therefore leads to a more cost efficient solution in case of link-by-link grooming and therefore a better position of this scenario so that OXC introduction becomes interesting only later on. The right most part of Fig. 15 shows that the expenses going to unequipped OXCs are relatively high in case of small traffic demands (until 2006), which explains why OXC introduction is not beneficial in this case. The middle part of Fig. 15 clearly illustrates that the use of island based grooming avoids the high costs for OXCs in case of small traffic demand as well as the high costs for SDH line cards in case of big traffic demands. Note, finally, that the relative expenses going to the different component types are more or less stable from 2010 onwards, because the granularity of the components fits the traffic demands rather well.

As explained before, in case of island based grooming, the dimensioning of a certain node is calculated according to both approaches (link-by-link and end-toend grooming) and the solution of this cost trade-off determines whether or not to introduce the OXC. As the equipment costs can influence this trade-off, they also influence the island evolution. Figure 16 indicates the number of end-to-end grooming nodes installed according to the island based grooming approach, in case of 
Fig. 14 Capex comparison between grooming approaches, based cumulative equipment costs
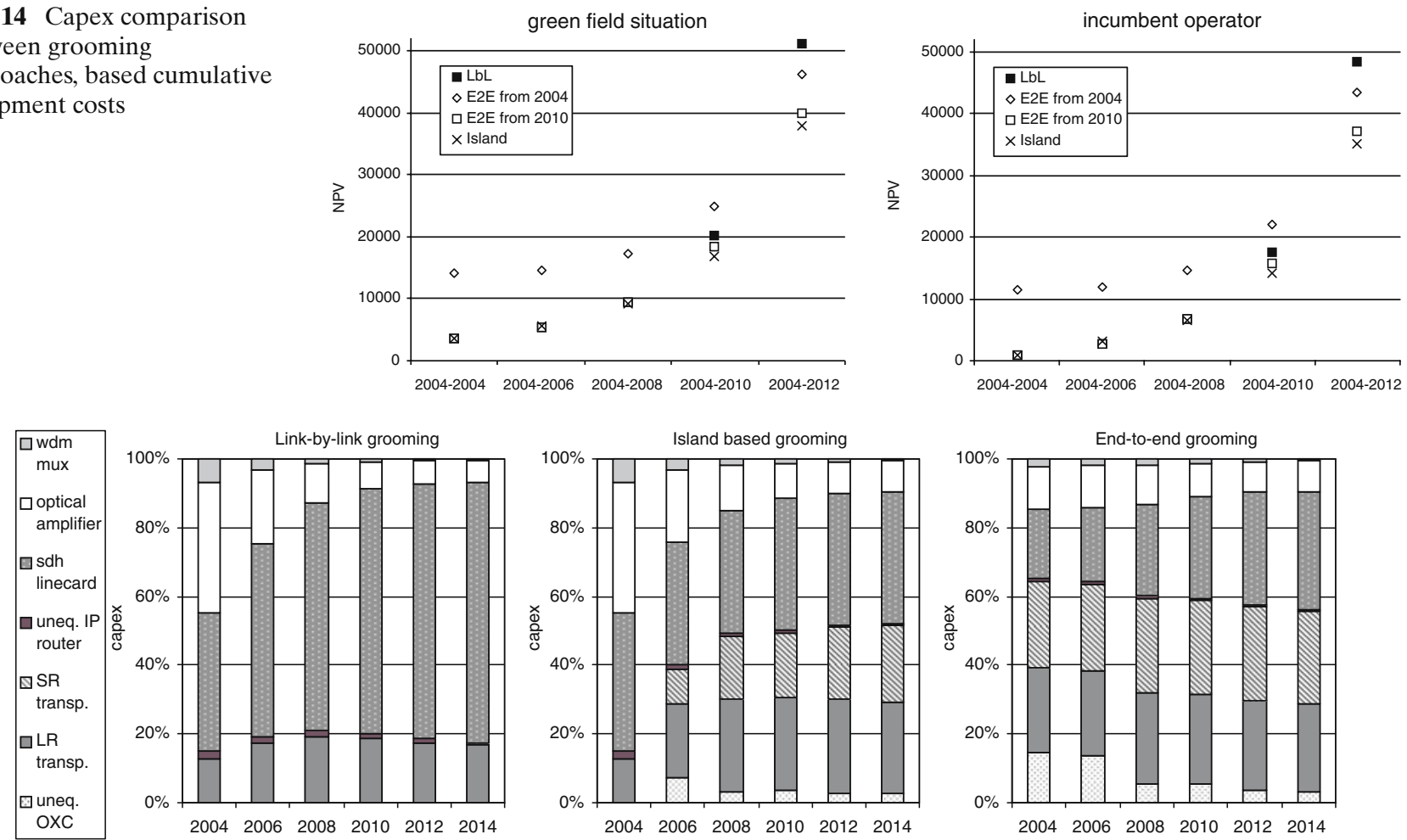

Fig. 15 Cost distribution over time for several grooming approaches (original cost figures)

reduced equipment costs (to half of their original value). The graph legend indicates which equipment type has a reduced cost in which curve (the other costs are unaffected). In case no component type has a reduced cost, we find the results already shown in Table 1 . The number of end-to-end grooming nodes evolves from 0 to 20 over the time period 2004-2014. Exactly the same evolution is found in case of reduced WDM line system or optical amplifier costs. With reduced SDH line card costs, far less OXCs are installed. Until 2006 no OXCs are used, in 2008 they become beneficial in ten nodes and for 2014 we find only 16 end-to-end grooming nodes. It is clear that a reduced SDH line card cost favours the use of linkby-link grooming (drastically reducing its cost) over the introduction of OXCs. A reduced transponder cost leads to the introduction of more end-to-end grooming nodes (up to 24 in 2014). Reducing the unequipped OXC cost only slightly favours the introduction of OXCs. The sensitivities found in Fig. 16 correspond to the observations of Table 1.

\section{Conclusions and guidelines}

The goal of this article was to compare several traffic grooming approaches, based on expected capex to dimension and expand the network. When making a trade-off between the traditional link-by-link approach

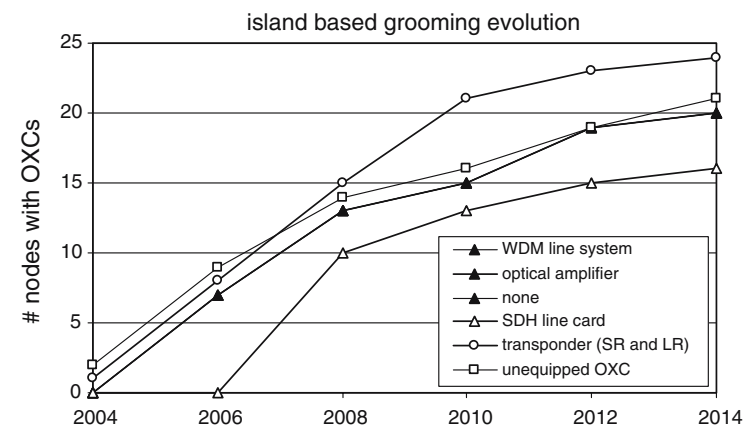

Fig. 16 Sensitivity of equipment costs on island evolution (curves for original costs, reduced WDM line system cost and reduced optical amplifier cost coincide)

and the use of end-to-end grooming, with OXCs in all nodes, we observe that, for small traffic demands, the link-by-link grooming approach (efficiently filling SDH frames) is most interesting. As traffic increases, however, there comes a point where the savings in IP layer expenses realized by end-to-end grooming compensate the extra expenses of introducing the needed OXCs. In practice, however, it is very unlikely that the migration from link-by-link towards end-to-end grooming happens at a single point in time. Therefore, we also studied a possible migration path, where OXCs are introduced gradually in so-called end-to-end grooming islands. Simulations have shown that this island based grooming approach can lead to important savings in capex. In order to study 
the realistic case of an incumbent operator, we did not only consider total costs, but also incremental and cumulative costs, indicating the total expenses to be paid by the operator over a certain time interval to extend his network. In this regard, the use of the NPV technique was clarified. Finally, we also studied the sensitivity of the obtained results to changes in the component costs. It was shown that a reduction in the SDH line card cost leads to less OXC introductions.

The following guidelines can be drawn from our work:

- To determine a reliable network expansion plan, it is important to estimate (current and future) network equipment costs as accurate as possible. If actual cost figures are impossible to obtain, relative figures indicating the most important trends can already give a lot of valuable information. Especially the costs of SDH line cards, SR and LR transponders and unequipped OXCs have an important impact on the cost comparison between the different grooming approaches.

- The main cost driver in case of link-by-link grooming is the SDH line card costs. A lower line card cost favours the use of link-by-link grooming, whereas a higher cost favours the introduction of OXCs and the use of end-to-end grooming. It can therefore be interesting to thoroughly compare the SDH line card costs set by different vendors.

- With a growing traffic demand, introducing OXCs in the network and migrating towards end-to-end grooming will become beneficial. To be able to spread the expenses and gradually adapt to the growing traffic demand, the island based grooming approach is a straightforward and effective approach.

- For an incumbent operator, long-term investment decision should be based on incremental instead of total costs. When comparing different network solutions over a certain time frame, cumulative discounted costs are to be considered.

Acknowledgements This work has been financed by IWT-Flanders through a $\mathrm{PhD}$ grant for the first author and the GBOU-project IWT 010058. It was also supported be the IWT/ITEA TBones project, by Ghent University through the RODEO-project and by the European Commission through ISTNOBEL and ePhoton/One.

\section{References}

[1] Gupta, V. P. (1985). What is network planning? IEEE Communications Magazine, 23(10), 10-16

[2] King, W. R., \& Premkumar, G. (1989). Key issues in Telecommunications Planning. Information and Management, vol. 17. (pp. 255-265). Amsterdam: Elsevier North-Holland.
[3] Pickavet, M., \& Demeester, P. (2000). Multi-Period Planning of Survivable WDM Networks. European Transaction on Telecommunications special issue on WDM networks, 11(1), $7-16$.

[4] Odlyzko, A. (2003). Internet traffic growth: Sources and implications, Optical Transmission Systems and Equipment for WDM Networking II. Proceedings SPIE., 5247, $1-15$.

[5] Zhu, K., \& Mukherjee, B. (2003). A review of traffic grooming in WDM optical networks: architectures and challenges. Optical Networks Magazine, 4(2), 55-64.

[6] Geary, N., Antonopoulos, A., \& O'Reilly, J. (2003). Optical Cross Connect architectures and analysis-Analysis of the potential benefits of OXC-based intelligent optical networks. Optical Networks Magazine, 4(2), 20-31.

[7] Battiti, R., \& Brunato, M. (2001). Reactive search for traffic grooming in WDM networks, Proceedings of 4th International Workshop on Digital Communication (pp. 56-66).

[8] Konda, V. R., \& Chow, T. Y. (2001). Algorithm for traffic grooming in optical networks to minimize number of transceivers, Proceedings of IEEE Workshop on High Performance Switching and Routing.

[9] De Maesschalck, S., Pickavet, M., Colle, D., \& Demeester, P. (2003). Multi-layer traffic grooming in networks with an IP/MPLS layer on top of a meshed optical layer. Proceedings of Globecom 2003. San Francisco, USA, December 2003.

[10] Gerstel, O., Ramaswami, R., \& Sasaki, G. (1998). Cost effective traffic grooming in WDM rings. Proceedings of 17th Conference on Computer Communication (pp. 69-77). San Francisco, USA, April 1998.

[11] Kandula, R., \& Sasaki, G. (2001). Grooming of dynamic tributary traffic in WDM rings with rearrangements, Proceedings of 39th Allerton conference on communication. Control and Computing, Moticello, USA, October 2001.

[12] ITU-T Recommendation G.957. Optical interfaces for equipments and systems relating to the synchronous digital hierarchy.

[13] Derkacz, J., Leszczuk, M., Wajda, K., Leone, R., Monari, G., Lievens, I., De Maesschalck, S., Verbrugge, S., \& Collem, D. (2002). IP/OTN Cost Model and Photonic Equipment Forecast - IST Lion Project, Proceedings of Workshop on Telecommunication Techno-Economics (pp. 126-138). Rennes, France, May 2002.

[14] Olsen, B. T. (1995). Forecasts of cost components, Proceedings of RACE 2087/TITAN Workshop. (Aveiro, Portugal, July 1995).

[15] Ross, S. A., Westerfield, R. W., \& Jaffe, J. F. (2002). Corporate finance. McGraw-Hill.

[16] De Maesschalck, S., Colle, D., Lievens, I., Pickavet, M., Demeester, P., Mauz, C., Jaeger, M., Inkret, R., Mikac, B., \& Derkacz, J. (2003). Pan-European optical transport networks: an availability-based comparison. Photonic Network Communications, 5(3), 203-225.

[17] Vaughn, M., \& Wagner, R. (2000). Metropolitan network traffic demand study, Proceedings Of 13th annual meeting of the Lasers and Electro-Optics Society (LEOS 2000), vol. 1 (pp. 102-103). Rio Grande, Puerto Rico, November 2000.

[18] Vaughn, M., Chia, M., \& Wagner, R.: A bottom-up traffic demand model for long-haul metropolitan optical networks, Proceedings of opitcal fibre conference (OFC2003). vol. 1, MF111 (pp. 139-150). Atlanta, USA, March 2003.

[19] http://www.ibcn.intec.ugent.be/css_design/research/projects/ INTERNAL/NRS/index.html

[20] Skrimont, D. (2000). Bigger, badder, faster: Routers grew up. Telecommunications. (November 2000) 
[21] El-Bawab, T. S., Agrawai, A., Poppe, F., Sofman, LB., Papadimitriou, D., Rousseau, B. (2003). The evolution to optical switching-based core networks. Optical Networks Magazine, 4(2), 7-19.

[22] Verbrugge, S., Colle, D., Pickavet, M., \& Demeester, P. (2004). An economical view on using OXCs in a European backbone, Proceedings of OECC-COIN 2004 (pp. 406-407). Pacifico Yokohama, Japan, July 2004.

[23] Verbrugge, S., Colle, D., Pickavet, M., \& Demeester, P. (2004). Capex cost comparison between link-by-link and end-to-end grooming in a European backbone network, Proceedings of Networks 2004 conference (pp. 27-32). Vienna, Austria, June 2004.

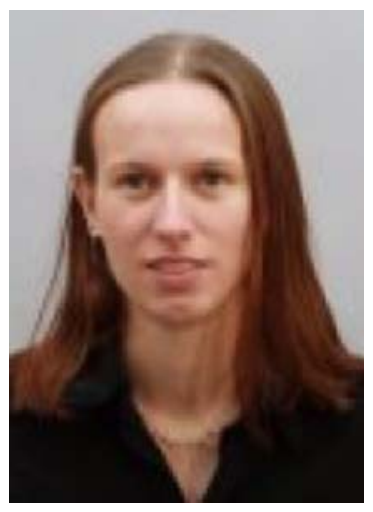

Sofie Verbrugge received a M.Sc. degree in computer science engineering from Ghent University (Ghent, Belgium) in 2001. She is currently working toward the Ph.D. degree in the IBCN-group of the Department of Information Technology, at the same university, as a researcher for the Institute for the Promotion of Science and Technology in Flanders (IWT-Vlaanderen), in the field of long-term planning of optical networks. Her main research interests include techno-economic aspects of telecom network planning, such as migration scenarios, investment decision techniques and cost modelling. During the summer of 2004, she was a visiting scientist at the Technologiezentrum of T-Systems (Deutsche Telekom, Berlin, Germany) where she mainly worked on the development of an operational cost model. She has been involved in the European IST-LION and IST-NOBEL projects as well as in the ITEA-TBONES project.

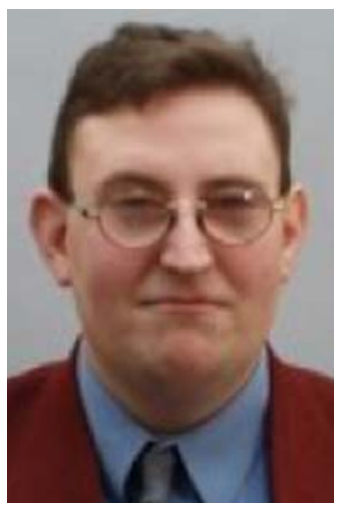

Didier Colle received a M. Sc. degree in electrotechnical engineering (option: communications) from the Ghent University in 1997. Since then, he has been working at the same university as researcher in the department of Information Technology (INTEC). He is part of the research group INTEC Broadband Communication Networks (IBCN) headed by prof: Piet Demeester. His research lead to a Ph.D degree in February 2002. He was granted a postdoctoral scholarship from the Institute for the Promotion of Innovation through Science and Technology in Flanders (IWT-Vlaanderen) in the period 2003-2004. His research deals with design and planning of communication networks. This work is focussing on optical transport networks, to support the nextgeneration Internet. Up till now, he has actively been involved in several IST projects (LION, OPTIMIST, DAVID, STOLAS, NOBEL and LASAGNE), in the COST-action 266 and 291 and in the ITEA/IWT TBONES project. His work has been published in more than 100 scientific publications in international conferences and journals.

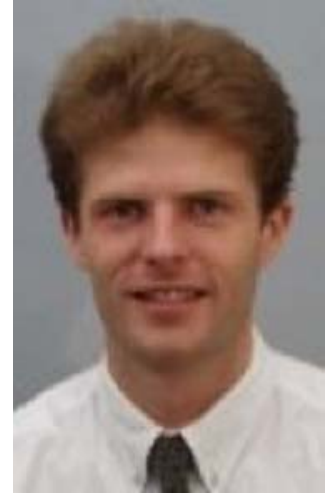

Mario Pickavet received a Master of Science degree and a Doctor of Electrical Engineering degree, specialized in telecommunications, from Ghent University in 1996 and 1999, respectively. Since 2000, he has been a full-time professor at the same university. His research interests are related to broadband communication networks (i.e., IP, MPLS WDM, SDH, ATM) and include resilience mechanisms, design, and long-term planning of core and access networks. In this context, he was and currently is involved in European IST projects (i.e. LION, DAVID, STOLAS, ePhoton/One, LASAGNE, NOBEL) on IP over WDM next generation networks. He has published a number of international publications on these subject, both in leading journals (e.g., IEEE Journal on Selected Areas in Communications and IEEE Communications Magazine) and proceedings of conferences. Together with JeanPhilippe Vasseur (Cisco) and Piet Demeester (Ghent University), he published the book Network Recovery in the Morgan Kaufmann series in networking.

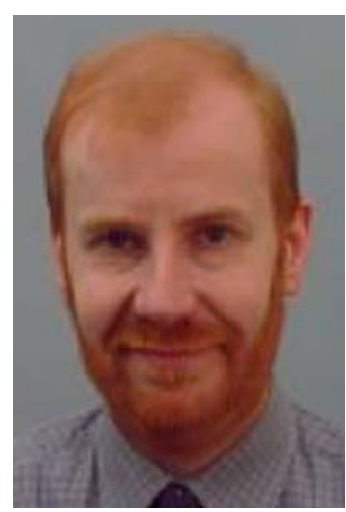

Piet Demeester is professor at Ghent University and head of the Broadband Communication Networks group (www.ibcn.intec.ugent.be). The group is part of the recently established Interdisciplinary institute for BroadBand Technology : IBBT (www.ibbt.be). His research interests include multilayer IP-Optical networks, mobile networks, end to end quality of service, grid computing, network and service management, distributed software and multimedia applications. He published over 500 papers in these areas in international journals and conference proceedings. 